\title{
A Comprehensive Prognostic Analysis of POLD1 in Hepatocellular Carcinoma
}

\author{
Hui Tang, Tingting You, Zhao Sun and Chunmei Bai* ${ }^{*}$
}

\begin{abstract}
Background: DNA polymerase delta 1 catalytic subunit (POLD1) plays a key role in DNA replication and damage repair. A defective DNA proofreading function caused by POLD1 mutation contributes to carcinogenesis, while POLD1 overexpression predicts poor prognosis in cancers. However, the effect of POLD1 in hepatocellular carcinoma (HCC) is not well-understood.

Methods: Expression patterns of POLD1 were evaluated in TCGA and the HPA databases. Kaplan-Meier curves and Cox regression were used to examine the prognostic value of POLD1. The prognostic and predictive value of POLD1 was further validated by another independent cohort from ICGC database. The influences of DNA copy number variation, methylation and miRNA on POLD1 mRNA expression were examined. The correlation between infiltrating immune cells and POLD1 expression was analyzed. GO and KEGG enrichment analyses were performed to detect biological pathways associated with POLD1 expression in HCC.

Results: POLD1 was overexpressed in HCC $(n=369)$ compared with adjacent normal liver $(n=50)$. POLD1 upregulation was significantly correlated with positive serum AFP and advanced TNM stage. Kaplan-Meier and multivariate analyses suggested that POLD1 overexpression predicts poor prognosis in HCC. DNA copy gain, low POLD1 methylation, and miR-139-3p downregulation were associated with POLD1 overexpression. Besides, POLD1 expression was associated with the infiltration levels of dendritic cell, macrophage, B cell, and CD4 + T cell in HCC. Functional enrichment analysis suggested "DNA replication", "mismatch repair" and "cell cycle" pathways might be involved in the effect of POLD1 on HCC pathogenesis. Additionally, POLD1 mRNA expression was significantly associated with tumor mutation burden, microsatellite instability, and prognosis in various tumors.
\end{abstract}

Conclusions: POLD1 may be a potential prognostic marker and promising therapeutic target in HCC.

Keywords: POLD1, Hepatocellular Carcinoma, The Cancer Genome Atlas database, Prognosis, Immunotherapy

\section{Background}

Hepatocellular carcinoma (HCC) is one of the most common primary tumors worldwide [1]. Unfortunately, the effect of drug treatment on HCC is limited, while the recurrence rate after surgery is about $70 \%$ at 5 years [1]. The World Health Organization speculated that more than 1 million patients will die of HCC in 2030 [1].

\footnotetext{
*Correspondence: baichunmei@pumch.cn

Department of Medical Oncology, Peking Union Medical College

Hospital, Chinese Academy of Medical Sciences, Peking Union Medical

College, 100730 Beijing, China
}

Therefore, it is urgent to further explore the underlying mechanisms of HCC carcinogenesis and development, which will contribute to the detection of novel promising prognostic and therapeutic targets.

DNA polymerase delta 1 catalytic subunit (POLD1) encodes the $125-\mathrm{kDa}$ catalytic subunit and provides the essential catalytic activity of DNA polymerase delta, which exhibits both DNA polymerase and 3' to 5' exodeoxyribonuclease activity, and plays a crucial role in DNA replication, DNA damage repair, cell growth and differentiation [2, 3]. Previous studies suggested that POLD1 was upregulated in HCC and breast cancer, and 
its overexpression correlated with tumor progression and poor prognosis $[4,5]$. Furthermore, POLD1 proofreading (exonuclease) domain mutations were associated with a deficient proofreading repair during DNA replication and an increased incidence of epithelial cancers, especially colorectal and endometrial cancer [6-8]. Recent studies demonstrated that POLD1 proofreading domain mutation can potentially predict desirable outcomes in cancer patients treated with immune-checkpoint inhibitors (ICIs) [9, 10]. However, the effect and underlying mechanisms of POLD1 in HCC are not well-understood.

In the present research, the comprehensive prognostic and predictive value of POLD1 in a well-defined HCC cohort from The Cancer Genome Atlas (TCGA) were first analyzed. The findings were further examined using an independent $\mathrm{HCC}$ cohort retrieved from International Cancer Genome Consortium (ICGC) database. The underlying mechanisms of POLD1 in HCC carcinogenesis and development were further analyzed by bioinformatics methods.

\section{Methods}

\section{Data source and processing}

The data (including clinical data, mRNA-seq data, and miRNA-seq data) of 369 primary HCC and 50 adjacent normal liver samples were downloaded from TCGALIHC (liver hepatocellular carcinoma) dataset in February 2021. After excluding those without complete Tumor-Node-Metastasis (TNM) stage and follow-up data, 339 HCC patients were enrolled. The validation cohort of 207 primary HCC and 175 adjacent normal liver samples were downloaded from ICGC-LIRI (liver cancer - RIKEN, Japan) dataset in March 2021. Somatic mutation data of TCGA pan-cancer cohort were downloaded from TCGA using UCSC Xena (http://xena.ucsc. edu) [11] in March 2021.

\section{Gene expression analysis}

POLD1 mRNA expression data in several different kinds of tumor tissues and normal controls were retrieved from GEPIA2 (http://gepia2.cancer-pku.cn), a handy tool to explore TCGA and GTEx datasets [12]. Immunohistochemistry (IHC) staining data of POLD1 in HCC and normal liver samples were retrieved from the Human Protein Atlas (HPA, http://www.proteinatlas.org) [13]. In the HPA database, protein expression rank was assessed by the staining intensity (strong/moderate/weak/negative) and fraction of stained cells $(>75 \% / 25-75 \% /<25 \%)$, including high (strong with $>25 \%$ ), medium (strong with $<25 \%$, or moderate with $>25 \%$ ), low (moderate with $<25 \%$, or weak with $>25 \%$ ), and not detected (weak or negative with $<25 \%$ ) [14].
The relationship between POLD1 mRNA expression and POLD1 DNA methylation or copy number alterations (CNA) in TCGA-LIHC were determined by cBioPortal for Cancer Genomics (http://www.cbioportal.org) [15] using TCGA-LIHC dataset. Moreover, the association between POLD1 mRNA expression and immune infiltrates in HCC was examined by TIMER2 (http://timer.cistrome.org) [16].

\section{Functional enrichment analysis}

The top 50 positively and negatively POLD1-correlated co-expression genes based on the TCGA-LIHC tumor tissues were retrieved from LinkedOmics (http://www. linkedomics.org) [17]. While the top 100 positively or negatively POLD1-correlated genes based on the TCGALIHC tumor and normal liver samples were retrieved from GEPIA2. The protein-protein interaction (PPI) network between POLD1 and other proteins, as well as 50 available experimentally determined POLD1-binding proteins, was retrieved from STRING (http://string-db. org) [18]. Furthermore, the GO (Gene ontology) enrichment analysis was conducted with the "clusterProfiler" package of $\mathrm{R}$ software using the combination of 100 POLD1-correlated genes and 50 POLD1-binding proteins. Besides, the KEGG (Kyoto encyclopedia of genes and genomes) enrichment analysis was performed by DAVID (https://david.ncifcrf.gov) [19] using the combination of the above two datasets. The enriched pathways were finally visualized with the "tidyr" and "ggplot2" packages of $R$ software.

\section{Tumor mutation burden and microsatellite instability in pan-cancer}

Tumor mutation burden (TMB) was defined as the total number of nonsynonymous mutations per megabase, and microsatellite instability (MSI) was calculated by the incidence of insertion or deletion that occurred in repeating sequences of genes. TMB scores were calculated with somatic mutation data of TCGA pan-cancer cohort using a Perl script, and adjusted by dividing by the total length of exons. MSI data were derived from previously published research [20]. The results of correlation analysis between POLD1 expression and TMB or MSI were presented as radar plots, generated using the "fmsb" package of R software.

\section{Statistical analyses}

Receiver operating characteristic (ROC) curve was applied to examine the prognostic value of POLD1 expression using the "ROCR" package of $\mathrm{R}$ software, and the area under the curve (AUC) was calculated by the "verification" package. The correlation between POLD1 mRNA expression and the clinicopathological variables was evaluated by Pearson's Chi-square test. POLD1 expression between 
different clinicopathological groups was evaluated by the "edgeR" package of $\mathrm{R}$ software using Quasi-likelihood F-test. Adjusted P value $<0.05$ and $\mid \log 2$ FC (fold change) $\mid$ $>1$ were considered to be statistically significant. Propensity-score matching (PSM) was performed to mitigate the influence of confounding factors. The propensity scores were calculated by the "MatchIt" package of R software using multivariable logistic regression based on age, gender, family history, and residual tumor after surgery. We matched propensity scores 1:1 with the nearest neighbor method without replacement by using a 0.02 calipers width. The overall survival (OS) and disease-free interval (DFI) between the low and high POLD1 expression groups were compared by Kaplan-Meier analysis. Cox regression analysis was performed by the "finalfit" package of $\mathrm{R}$ software to calculate the hazard ratios (HR) and confidence intervals (CI) of factors associated with OS and DFI in $\mathrm{HCC}$ patients. According to the result of multivariate Cox analysis, the nomogram was established by the "rms" package of $\mathrm{R}$ software and assessed by the calibration curves and concordance index (C-index). Two-tailed $\mathrm{P}$ value $<0.05$ was regarded to be statistically significant.

Strawberry Perl (version 5.30.0, http://strawberryperl. com) was applied to extract POLD1 expression data from downloaded datasets. All statistical analyses and visualization were conducted using $\mathrm{R}$ software (version 3.6.1, https://www.r-project.org/).

\section{Result}

\section{Increased POLD1 expression in HCC}

POLD1 mRNA expression in several tumors and normal controls was retrieved from GEPIA2 (Fig. $1 \mathrm{~A}$ ). In TCGA-LIHC dataset, POLD1 expression was significantly upregulated in HCC tissues $(n=369)$ compared with adjacent normal liver samples $(n=50)(P<0.0001)$ (Fig. 1B). The ROC curve (AUC $=0.927, P<0.0001)$ suggested POLD1 upregulation was related to the diagnosis of $\mathrm{HCC}$ (Fig. $1 \mathrm{C}$ ). Moreover, the IHC data retrieved from the HPA was used to examine the expression of POLD1 at protein level. While normal liver samples usually displayed low POLD1 staining (3/3), most HCC tissues displayed medium $(7 / 11)$ or high (3/11) POLD1 staining, which is mainly located in the nucleus (Fig. 1D, E).

\section{POLD1 overexpression correlated with HCC progression}

The clinicopathological variables of 339 primary HCC patients retrieved from TCGA-LIHC were given in Table 1. Patients were classified into two groups (low/ high) according to their POLD1 expression level and based on the optimal cut-off value of OS calculated by the "survminer" package of R software. As shown in Table 1; Fig. 2, POLD1 expression was not significantly associated with age, gender, Ishak score, Child - Pugh grade, family history of cancer, histologic grade, vascular invasion, and residual tumor (all $\mathrm{P}>0.05$ ). However, POLD1 upregulation was significantly correlated with positive alpha fetoprotein (AFP), and advanced TNM stage (all $P<0.05$ ).

\section{POLD1 overexpression predicted poor OS in HCC}

The result of univariate analysis demonstrated that POLD1 mRNA level, TNM stage, and vascular invasion were significantly associated with the OS of HCC patients (Table 2). Further multivariate analysis confirmed that POLD1 overexpression (HR: 1.47, 95\% CI: $1.1-1.97, P=0.001)$ and advanced TNM stage were independent indicators of unfavorable OS (Table 2). As shown in Table 3, the univariate analysis demonstrated that POLD1 mRNA level was associated with the DFI of HCC patients. However, the multivariate analysis determined that POLD1 upregulation was not an independent indicator of poor DFI (HR: 1.29, 95\% CI: 0.99-1.68, $P=0.057$ ) (Table 3). Furthermore, Kaplan-Meier curves and log-rank tests were performed to show the effect of POLD1 expression on the OS and DFI of unpaired HCC patients (Fig. $3 \mathrm{~A}, \mathrm{~B}$ ) and matched $\mathrm{HCC}$ patients after PSM analysis (Fig. 3 C, D).

\section{Validation of the prognostic value of POLD1 based on nomogram}

Based on the result of multivariate Cox analyses (Table 2), the nomogram predicting the OS of HCC patients was constructed based on TNM stage and POLD1 mRNA expression level (Fig. 4 A). The C-index of the nomogram for OS prediction was 0.655 (95\% CI: 0.627-0.684). As shown in the calibration plot (Fig. 4B), the nomogram demonstrated decent agreement between the predicted and actual survival outcome (1-, 3-, and 5-year OS). Besides, the AUC for 1-, 3-, and 5-year OS were 0.697, 0.734, and 0.694, respectively (Fig. 4 C).

\section{Validation of the prognostic value and clinical correlation of POLD1 using ICGC dataset}

The predictive and prognostic value of POLD1 were examined in an independent external HCC dataset from the ICGC. Patients were classified into two groups (low/ high) based on the median expression value of POLD1. As shown in Supplementary Figure S1A-D, the POLD1 mRNA was over-expressed in HCC samples $(n=207)$ compared with normal controls $(n=175)$ in ICGC-LIRI dataset. Moreover, POLD1 overexpression was correlated with advanced TNM stage and higher histologic grade. The Kaplan-Meier curve and log-rank test suggested POLD1 expression predicted poor OS in HCC patients from independent ICGC-LIRI dataset $(P=0.004)$ (Supplementary Figure S2). 




DNA copy gain, low POLD1 methylation and miR139-3p downregulation contributed to POLD1 overexpression in $\mathrm{HCC}$

The underlying mechanisms of POLD1 overexpression in $\mathrm{HCC}$ carcinogenesis and development were further explored in terms of genetic and epigenetic alterations. There were 364 HCC patients with complete mRNA and CNA data in TCGA database, three patients had POLD1 amplification and 72 patients had POLD1 copy gain (low-level amplification). As shown in Fig. 5 A, 
Table 1 Association between POLD1 expression and the clinicopathological variables in HCC patients $(n=339)$

\begin{tabular}{|c|c|c|c|}
\hline \multirow[t]{2}{*}{ Variables } & \multicolumn{2}{|c|}{ POLD1 Expression } & \multirow[t]{2}{*}{$P$} \\
\hline & High $(n=172)$ & Low $(n=167)$ & \\
\hline \multicolumn{4}{|l|}{ Age (year) } \\
\hline$<65$ & $112(65.1 \%)$ & $96(57.5 \%)$ & \multirow[t]{2}{*}{0.183} \\
\hline$\geq 65$ & $60(34.9 \%)$ & $71(42.5 \%)$ & \\
\hline \multicolumn{4}{|l|}{ Gender } \\
\hline Male & $112(65.1 \%)$ & $119(71.3 \%)$ & \multirow[t]{2}{*}{0.273} \\
\hline Female & $60(34.9 \%)$ & $48(28.7 \%)$ & \\
\hline \multicolumn{4}{|c|}{ Family history of cancer } \\
\hline No & $104(60.5 \%)$ & $92(55.1 \%)$ & \multirow[t]{3}{*}{0.153} \\
\hline Yes & $42(24.4 \%)$ & $56(33.5 \%)$ & \\
\hline Unknown & $26(15.1 \%)$ & $19(11.4 \%)$ & \\
\hline \multicolumn{4}{|l|}{ TNM stage } \\
\hline । & $71(41.3 \%)$ & 99 (59.3\%) & \multirow[t]{4}{*}{$<0.001$} \\
\hline$\|$ & $47(27.3 \%)$ & $37(22.2 \%)$ & \\
\hline III & $54(31.4 \%)$ & $27(16.2 \%)$ & \\
\hline IV & $0(0 \%)$ & $4(2.4 \%)$ & \\
\hline \multicolumn{4}{|c|}{ Histologic grade } \\
\hline G1-G2 & $88(51.2 \%)$ & $124(74.3 \%)$ & \multirow[t]{3}{*}{$<0.001$} \\
\hline G3-G4 & $83(48.3 \%)$ & $42(25.1 \%)$ & \\
\hline Unknown & $1(0.6 \%)$ & $1(0.6 \%)$ & \\
\hline \multicolumn{4}{|l|}{ Ishak score } \\
\hline $0-4$ & $61(35.5 \%)$ & $63(37.7 \%)$ & \multirow[t]{3}{*}{0.013} \\
\hline $5-6$ & $28(16.3 \%)$ & $46(27.5 \%)$ & \\
\hline Unknown & $83(48.3 \%)$ & $58(34.7 \%)$ & \\
\hline \multicolumn{4}{|c|}{ Child-Pugh grade } \\
\hline A & $103(59.9 \%)$ & $104(62.3 \%)$ & \multirow[t]{3}{*}{0.331} \\
\hline $\mathrm{B}-\mathrm{C}$ & $8(4.7 \%)$ & $13(7.8 \%)$ & \\
\hline Unknown & $61(35.5 \%)$ & $50(29.9 \%)$ & \\
\hline \multicolumn{4}{|c|}{ Vascular invasion } \\
\hline None & $93(54.1 \%)$ & $100(59.9 \%)$ & \multirow[t]{4}{*}{0.230} \\
\hline Micro & $41(23.8 \%)$ & $43(25.7 \%)$ & \\
\hline Macro & $7(4.1 \%)$ & $7(4.2 \%)$ & \\
\hline Unknown & $31(18.0 \%)$ & $17(10.2 \%)$ & \\
\hline \multicolumn{4}{|c|}{ Alpha fetoprotein } \\
\hline Negative & $49(28.5 \%)$ & $94(56.3 \%)$ & \multirow[t]{3}{*}{$<0.001$} \\
\hline Positive & $82(47.7 \%)$ & $38(22.8 \%)$ & \\
\hline Unknown & $41(23.8 \%)$ & $35(21.0 \%)$ & \\
\hline \multicolumn{4}{|c|}{ Residual tumor } \\
\hline RO & $151(87.8 \%)$ & $150(89.8 \%)$ & \multirow[t]{3}{*}{0.761} \\
\hline$R 1-R 2$ & $6(3.5 \%)$ & $6(3.6 \%)$ & \\
\hline Unknown & 15 (8.7\%) & $11(6.6 \%)$ & \\
\hline
\end{tabular}

POLD1 amplification and copy gain was significantly correlated with POLD1 mRNA upregulation (all $P<0.05$ ). Besides, we examined the association between POLD1 DNA methylation and its mRNA expression. As shown in Fig. 5B, linear regression analysis demonstrated a significant negative correlation between POLD1 DNA methylation level and its mRNA expression (Pearson's $\mathrm{r}=-0.3, P<0.001)$.

Furthermore, we attempted to detect the regulatory miRNAs of POLD1. DIANA-microT database (http:// diana.imis.athena-innovation.gr) was used to detect the regulatory miRNAs of POLD1 [21]. The common miRNAs detected by DIANA-microT and confirmed by TCGA-LIHC dataset were regarded as the potential regulatory miRNAs of POLD1 in HCC. As a result, miR-139-3p, miR-214-3p, miR-5589-3p, miR-195-5p, and miR-424-5p were considered as the potential regulatory miRNAs. MiR-214-3p, miR-5589-3p, miR-195-5p, and miR-424-5p expression were not associated with OS and/or DFI in HCC patients (data not shown). However, Kaplan-Meier curves suggested that low miR-139-3p expression was related to poor OS and DFI in patients from TCGA-LIHC dataset (all $P<0.001$ ) (Fig. 5E, F). Therefore, miR-139-3p was selected for further exploration. As shown in Fig. $5 \mathrm{C}$, there was a significant negative correlation between the miR-139-3p and POLD1 mRNA expression (Pearson's $r=-0.54, P<0.001$ ). Compared to adjacent normal liver samples, miR-139-3p was significantly downregulated in HCC tissues $(P<0.001)$ (Fig. 5D). The putative binding site of POLD1 3' UTR by miR-139-3p retrieved from DIANA-microT was shown in Fig. 5G.

\section{POLD1 expression and immune cells infiltration analyses}

We also explored the association between POLD1 expression and immune cell infiltration in HCC tissues. As shown in Fig. 6, there were significant positive correlations between POLD1 mRNA expression and immune infiltration level of B cell (Pearson's $r=0.468, P<0.001$ ), CD4 $+\mathrm{T}$ cell (Pearson's $\mathrm{r}=0.358, P<0.001$ ), macrophage (Pearson's $r=0.397, P<0.001$ ), and dendritic cell (Pearson's $\mathrm{r}=0.438, \quad P<0.001)$. However, the correlation between POLD1 expression and CD8 + T Cell infiltration level (Pearson's $r=0.277, P<0.001$ ) was weak.

\section{Co-expression genes and PPI network of POLD1}

Genes co-expressed with POLD1 were retrieved from LinkedOmics, which analyzed mRNA sequencing data of 371 patients from TCGA-LIHC dataset. The heatmaps (Fig. 7 A, B) showed the top 50 significant genes positively and negatively correlated with POLD1. The volcano plot (Fig. $7 \mathrm{C}$ ) showed all the genes associated with POLD1. While WDR62, CDT1 and MCM2 were the top three genes positively correlated with POLD1 mRNA expression (Fig. 8 A-C), MMAA, SUCLG2 and CBR4 were the top three genes negatively correlated with POLD1 mRNA expression (Fig. 8D-F). Moreover, we performed Pearson correlation analysis to examine the relationship 


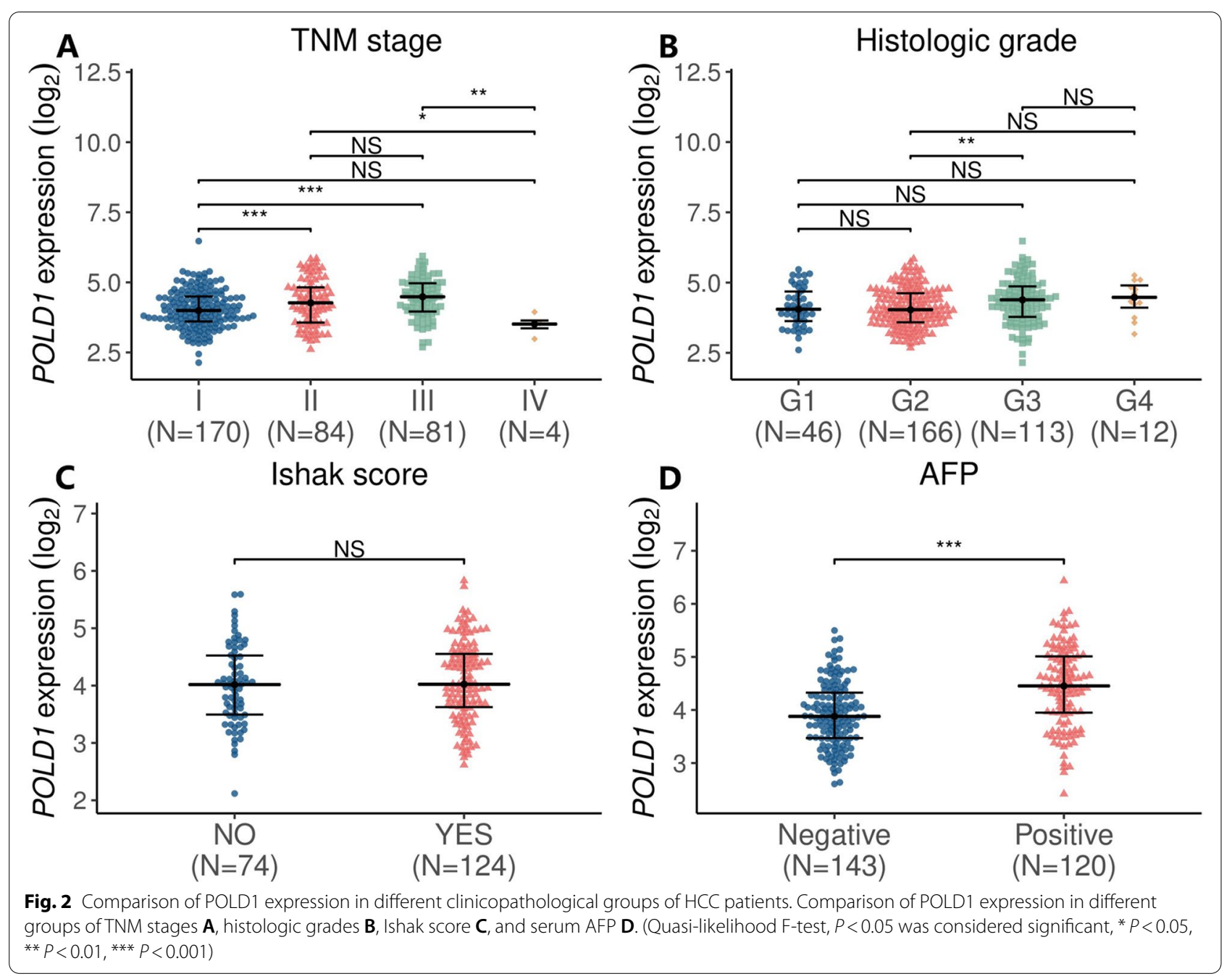

Table 2 Univariate and multivariate analyses of overall survival

\begin{tabular}{|c|c|c|c|c|}
\hline \multirow[t]{2}{*}{ Variables } & \multicolumn{2}{|c|}{ Univariate analysis } & \multicolumn{2}{|c|}{ Multivariate analysis } \\
\hline & HR $(95 \%$ Cl) & $\mathbf{P}$ & HR $(95 \% \mathrm{Cl})$ & $\mathbf{P}$ \\
\hline Age $(\geq 65$ vs. $<65)$ & $1.23(0.85,1.78)$ & 0.273 & - & - \\
\hline Gender (Female vs. Male) & $1.26(0.87,1.84)$ & 0.228 & - & - \\
\hline Family history of cancer (Yes vs. No) & $1.14(0.76,1.69)$ & 0.53 & - & - \\
\hline TNM stage (II vs. I) & $1.42(0.87,2.32)$ & 0.16 & $1.21(0.64,2.28)$ & 0.556 \\
\hline TNM stage (III vs. I) & $2.72(1.78,4.15)$ & $<0.001$ & $1.91(1.11,3.3)$ & 0.02 \\
\hline TNM stage (IV vs. I) & $5.44(1.68,17.63)$ & 0.005 & $6.57(2,21.63)$ & 0.002 \\
\hline Histologic grade (G3-G4 vs. G1-G2) & $1.14(0.78,1.67)$ & 0.489 & - & - \\
\hline Ishak score (5-6 vs. 0-4) & $0.87(0.5,1.5)$ & 0.612 & - & - \\
\hline Child-Pugh grade (B-C vs. A) & $1.66(0.82,3.36)$ & 0.159 & - & - \\
\hline Vascular invasion (Micro vs. None) & $1.16(0.72,1.88)$ & 0.539 & $1(0.57,1.75)$ & 0.989 \\
\hline Vascular invasion (Macro vs. None) & $2.52(1.14,5.58)$ & 0.023 & $2.02(0.88,4.62)$ & 0.097 \\
\hline Alpha fetoprotein (Positive vs. Negative) & $1.45(0.92,2.28)$ & 0.108 & - & - \\
\hline Residual tumor (R1-R2 vs. R0) & $1.17(0.43,3.2)$ & 0.754 & - & - \\
\hline POLD1 (High vs. Low) & $1.58(1.23,2.02)$ & $<0.001$ & $1.47(1.1,1.97)$ & 0.01 \\
\hline
\end{tabular}


Table 3 Univariate and multivariate analyses of disease-free interval

\begin{tabular}{|c|c|c|c|c|}
\hline \multirow[t]{2}{*}{ Variables } & \multicolumn{2}{|c|}{ Univariate analysis } & \multicolumn{2}{|c|}{ Multivariate analysis } \\
\hline & $\mathrm{HR}(95 \% \mathrm{Cl})$ & $\mathbf{P}$ & $\mathrm{HR}(95 \% \mathrm{Cl})$ & $\mathbf{P}$ \\
\hline Age $(\geq 65$ vs. $<65)$ & $1.09(0.76,1.54)$ & 0.65 & - & - \\
\hline Gender (Female vs. Male) & $0.88(0.61,1.27)$ & 0.487 & - & - \\
\hline Family history of cancer (Yes vs. No) & $0.92(0.63,1.36)$ & 0.683 & - & - \\
\hline TNM stage (II vs. I) & $1.7(1.12,2.6)$ & 0.014 & $1.78(1.05,3.02)$ & 0.032 \\
\hline TNM stage (III vs. I) & $2.84(1.89,4.26)$ & $<0.001$ & $2.73(1.65,4.52)$ & $<0.001$ \\
\hline TNM stage (IV vs. I) & - & - & - & - \\
\hline Histologic grade (G3-G4 vs. G1-G2) & $1.26(0.89,1.77)$ & 0.197 & - & - \\
\hline Ishak score (5-6 vs. 0-4) & $1.32(0.87,2.01)$ & 0.196 & - & - \\
\hline Child-Pugh grade (B-C vs. A) & $1.43(0.74,2.76)$ & 0.287 & - & - \\
\hline Vascular invasion (Micro vs. None) & $1.35(0.89,2.05)$ & 0.161 & $0.92(0.56,1.5)$ & 0.735 \\
\hline Vascular invasion (Macro vs. None) & $2.82(1.34,5.92)$ & 0.006 & $2.4(1.12,5.18)$ & 0.025 \\
\hline Alpha fetoprotein (Positive vs. Negative) & $1.08(0.74,1.59)$ & 0.691 & - & - \\
\hline Residual tumor (R1-R2 vs. R0) & - & - & - & - \\
\hline POLD1 (High vs. Low) & $1.36(1.08,1.71)$ & 0.01 & $1.29(0.99,1.68)$ & 0.057 \\
\hline
\end{tabular}

between POLD1 and POLD molecular family, as well as DNA polymerase epsilon (POLE) (Fig. 7D). Furthermore, we obtained 50 POLD1-binding proteins from STRING, which were supported by experimental evidence. Figure 7E showed the interaction network of these proteins. Figure $7 \mathrm{~F}$ showed the intersection analysis of the top 100 POLD1-correlated genes retrieved from GEPIA2 and 50 POLD1-binding proteins obtained from STRING, demonstrating the three common members (CDC45, POLA2 and CHTF18) among the above two datasets.

\section{Functional enrichment analysis of POLD1 in HCC}

GO and KEGG pathway enrichment analyses were performed with the combination of 100 POLD1-correlated genes and 50 POLD1-binding proteins. The GO analysis demonstrated that most of these genes were linked to the pathways or cellular biology of DNA replication (such as helicase activity, DNA polymerase activity), DNA damage repair (such as damaged DNA binding) and DNA metabolism (such as catalytic activity acting on) (Fig. 9 A, B). The KEGG analysis demonstrated "DNA replication", "nucleotide excision repair" and "cell cycle" pathways might be involved in the effect of POLD1 on HCC pathogenesis (Fig. 9 C).

\section{Effect of POLD1 mRNA expression on prognosis and genomic stability in pan-cancer}

We further evaluated the effect of POLD1 mRNA expression on the prognosis of patients with various cancer types. To simplify the research work, patients were classified into two groups (low/high) based on the median expression value of POLD1. As shown in
Supplementary Figure S3A, univariate Cox analysis suggested that POLD1 mRNA level was associated with the OS of patients with adrenocortical carcinoma (ACC, HR: 5.31, 95\% CI: 2.84-9.93, $P<0.001)$, diffuse large B-cell lymphoma (DLBC, HR: 0.18, 95\% CI: 0.03-0.99, $P=0.049$ ), kidney chromophobe (KICH, HR: 5.79, 95\% CI: $1.45-23.13, P=0.013)$, clear cell renal cell carcinoma (KIRC, HR: 2.46, 95\% CI: 1.73-3.51, $P<0.001$ ), brain lower grade glioma (LGG, HR: 1.93, 95\% CI: 1.53-2.44, $P<0.001$ ), mesothelioma (MESO, HR: 2.97, 95\% CI: 1.87-4.72, $P<0.001$ ), pheochromocytoma and paraganglioma (PCPG, HR: 9.25, 95\% CI: 1.47-58.34, $P=0.018$ ), prostate adenocarcinoma (PRAD, HR: 3.65, 95\% CI: 1.33-10, $P=0.012$ ), sarcoma (SARC, HR: $1.68,95 \% \mathrm{CI}$ : 1.22-2.31, $P=0.001$ ), uveal melanoma (UVM, HR: 0.11, 95\% CI: $0.02-0.46, P=0.003$ ), and HCC (HR: 1.65 , 95\% CI: $1.29-2.11, P<0.001)$. Moreover, POLD1 mRNA level was significantly associated with the DFI of patients with PRAD (HR: 4.73, 95\% CI: 2.14-10.41, $P<0.001$ ), SARC (HR: $1.61,95 \% \mathrm{CI}: 1.12-2.32, P=0.011)$, and HCC (HR: 1.29, 95\% CI: 1.06-1.58, $P=0.011$ ) (Supplementary Figure S3B). However, Kaplan-Meier curve and log-rank test suggested there was no significant association between POLD1 expression and the DFI of HCC $(P=0.078)$. Kaplan-Meier curves and log-rank tests were performed to show the effect of POLD1 expression on the OS and DFI of patients with various cancer types (Supplementary Figure S4 and S5).

TMB and MSI are considered to be important factors impacting carcinogenesis and survival. We determined the relationship between TMB or MSI and POLD1 mRNA expression in pan-cancer. The results suggested 


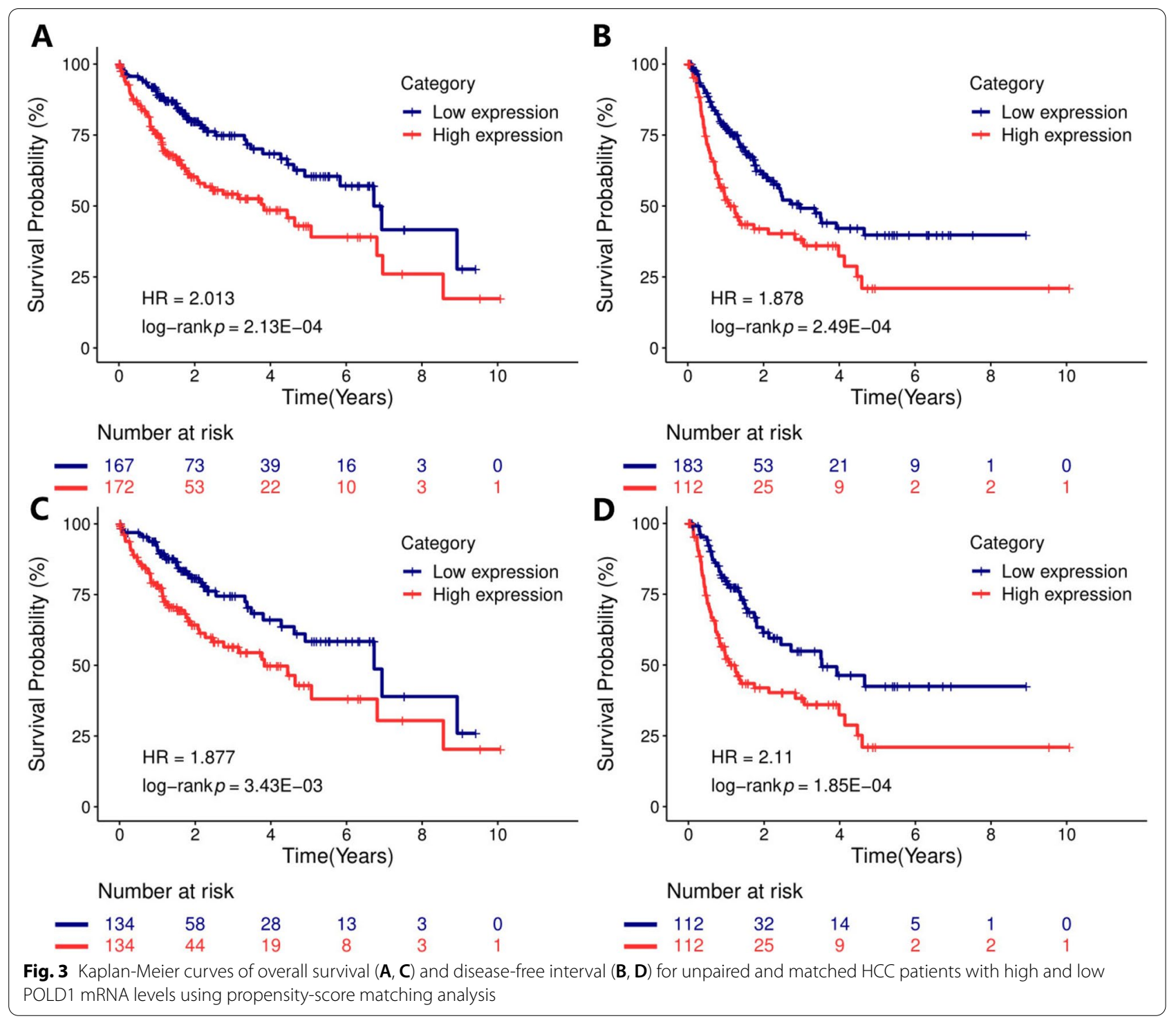

that POLD1 expression was significantly positively correlated with TMB in ACC, bladder urothelial carcinoma (BLAC), breast invasive carcinoma (BRCA), glioblastoma multiforme (GBM), KICH, LGG, lung adenocarcinoma (LUAD), lung squamous cell carcinoma (LUSC), MESO, ovarian serous cystadenocarcinoma (OV), pancreatic adenocarcinoma (PAAD), SARC, skin cutaneous melanoma (SKCM), stomach adenocarcinoma (STAD), testicular germ cell tumor (TGCT), thyroid carcinoma (THCA), uterine corpus endometrial carcinoma (UCEC), and uterine carcinosarcoma (UCS) (Supplementary Figure S3C). While increased POLD1 expression was significantly associated with decreased TMB in thymoma (THYM) (Supplementary Figure S3C). Likewise, POLD1 expression was significantly positively correlated with MSI in ACC, BLCA, BRCA, CESC, head and neck squamous cell carcinoma (HNSC), KICH, KIRC, HCC, LUAD, LUSC, PRAD, SARC, STAD, THCA, and UCEC (Supplementary Figure S3D). While increased POLD1 expression was significantly associated with decreased TMB in rectum adenocarcinoma (READ) (Supplementary Figure S3D).

\section{Discussion}

HCC accounts for approximately $80 \%$ of liver cancers, which is the sixth most common cancer and the fourth leading cause of cancer-related death worldwide [22]. With a 5 -year survival of $18 \%$ [1], it is needed to detect more novel diagnostic and therapeutic targets of $\mathrm{HCC}$, 


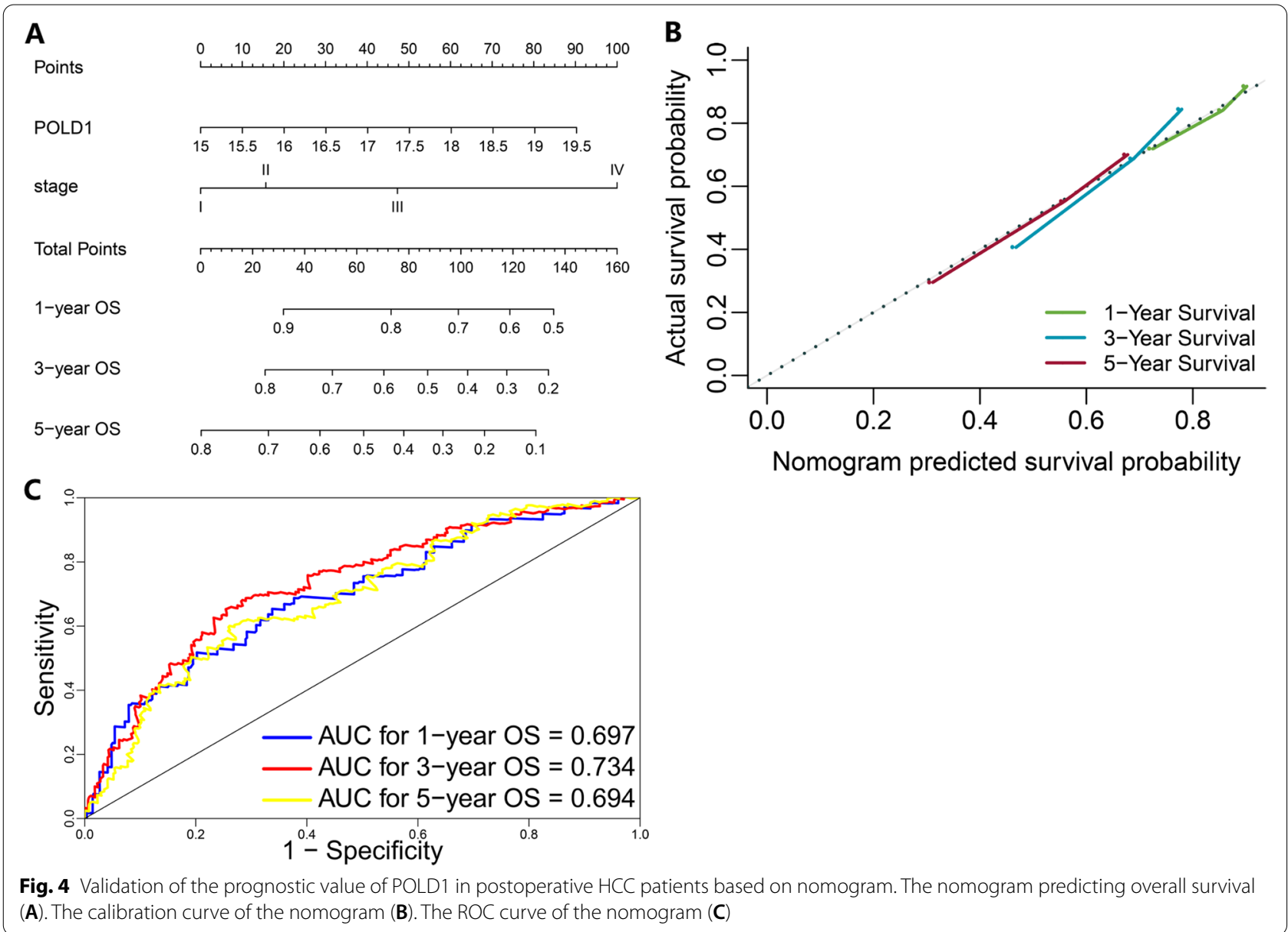

which will promote early diagnosis and personalized tumor management.

POLD1 plays a key role in cell cycle and DNA repair [23]. Previous studies suggested that POLD1 proofreading domain mutations were identified as predisposing to a range of cancers, including colorectal cancer, endometrial cancer, skin squamous cell carcinoma, breast cancer, and brain tumor [6-8]. Moreover, POLD1 upregulation contributes to cancer cell proliferation, migration, and invasion, as well as survival under replication stress via improving their tolerance to DNA damage [23, 24]. It has been proposed that an elevated level of DNA polymerase delta may favor late-stage oncogenesis [23].

Although studies have confirmed that ICIs-containing treatment regimens can improve the survival of patients with HCC, not all patients can benefit from ICIs [25]. Therefore, patient selection using predictive biomarkers would be desirable, and it has been proved that TMB and MSI status may be promising biomarkers [25]. Considering the role of POLD1 in DNA repair, it is understandable that POLD1 proofreading domain mutations, even non-proofreading mutations, are significantly correlated with higher TMB and high MSI (MSI-H) status $[9,10]$. Higher TMB and MSI-H status were believed to be associated with improved survival in patients treated with ICIs across multiple cancer types [26, 27]. Further, Wang et al. [10] suggested that POLE/POLD1 mutations could potentially predict desirable outcomes in patients treated with ICIs across multiple cancer types, even if cancer type and MSI status were adjusted. Moreover, the defective POLD1 function caused by its mutation has been regarded as a cause of $\mathrm{T}$ cell immunodeficiency [28]. Intriguingly, siRNA-mediated POLD1 depletion impaired proliferation and/or invasive potential, and increased genome instability of (liver, breast, cervical and osteosarcoma) cancer and normal cells [5, $29,30]$, which confirmed the key role of POLD1 in cell cycle progression and DNA damage repair. The relationship between two genes, in which single mutations alone are compatible with cell survival, but mutation of both leads to death, is defined as synthetic lethality [31]. Based on this concept, multiple studies demonstrated that colorectal cancer with deficient POLD1 activity possessed the increased sensitivity to ATR and CHK1 

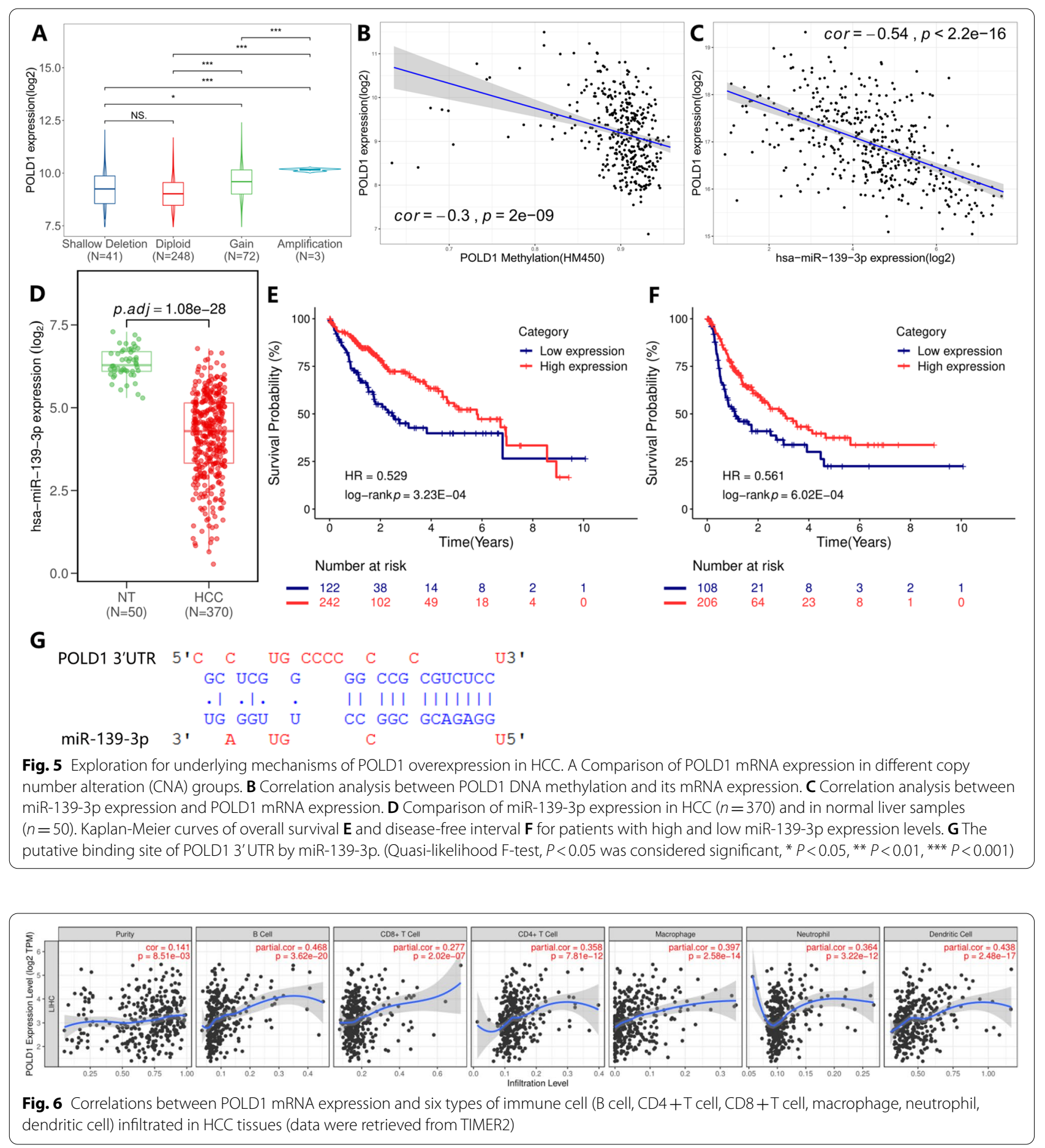

inhibitors in preclinical models [32, 33]. However, the effect and underlying mechanisms of POLD1 in HCC are not well-understood.

In the current study, using data from TCGA-LIHC and the HPA, we observed that POLD1 was over-expressed in HCC than in normal liver samples at mRNA and protein levels, which was verified by another independent research [5]. Moreover, POLD1 upregulation was an independent indicator of poor OS, but not DFI, and was correlated with positive AFP and advanced TNM stage. These findings, including the association between POLD1 mRNA expression and TNM stage, as well as the 

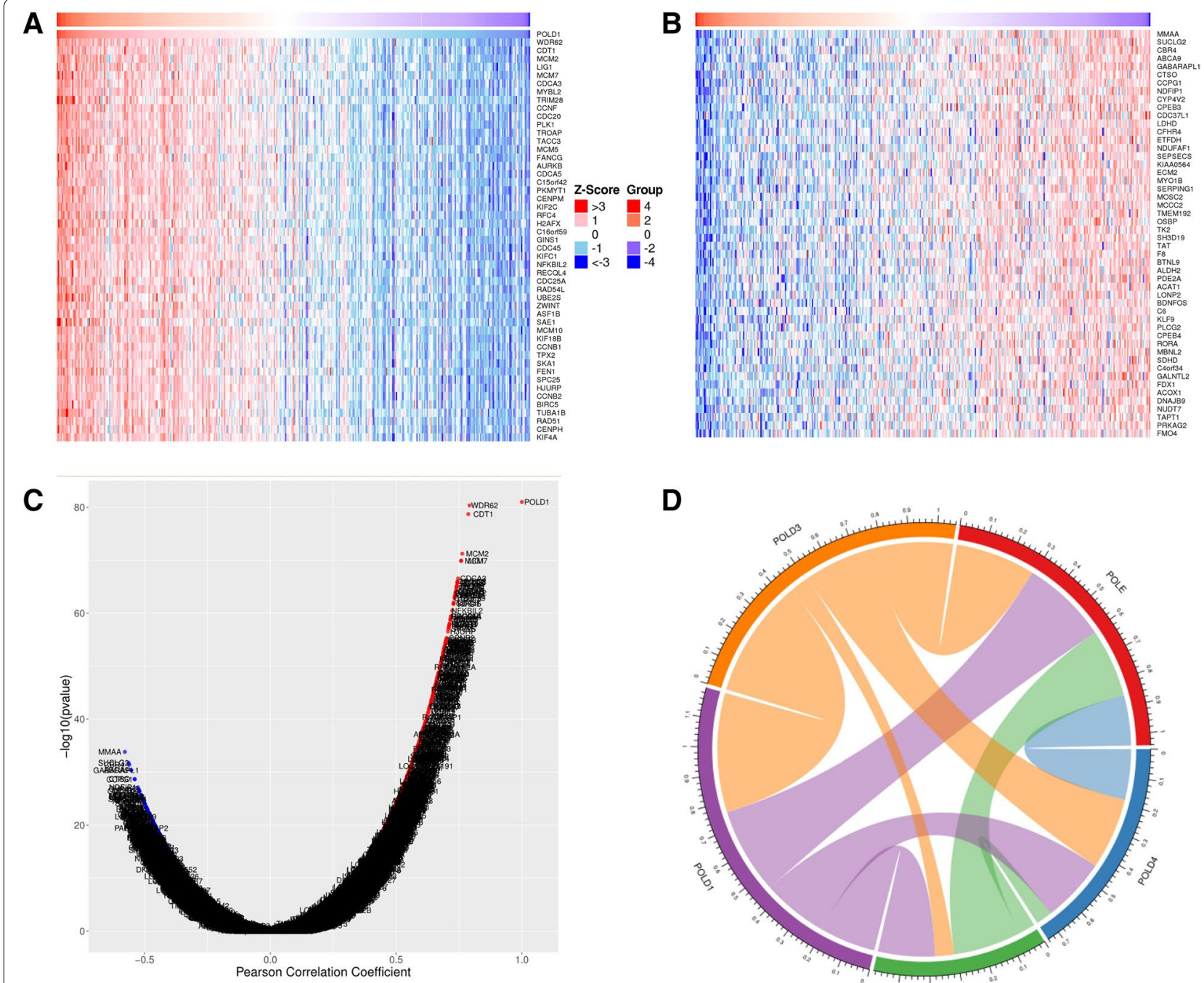

$\mathbf{E}$
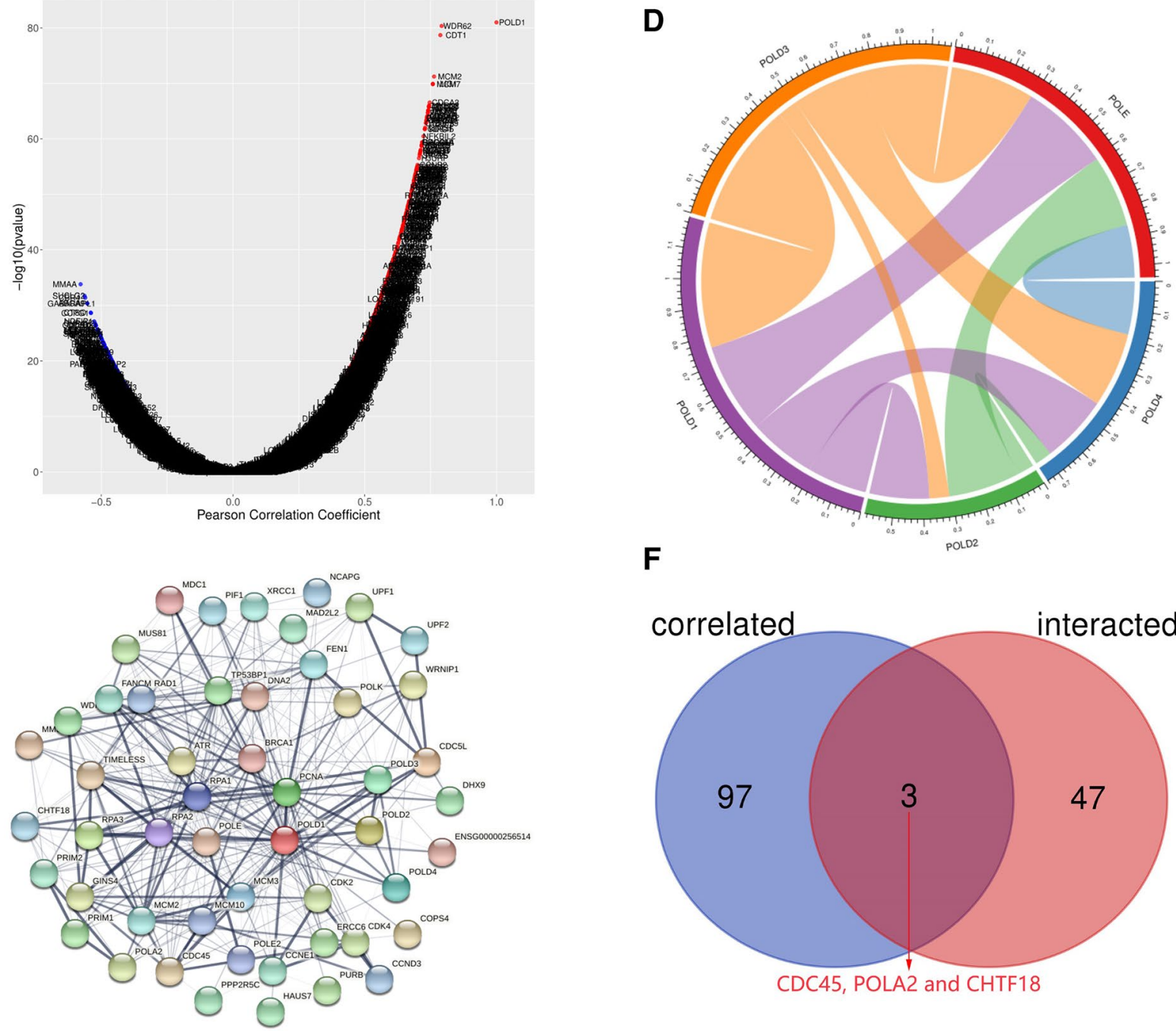

$\mathbf{F}$

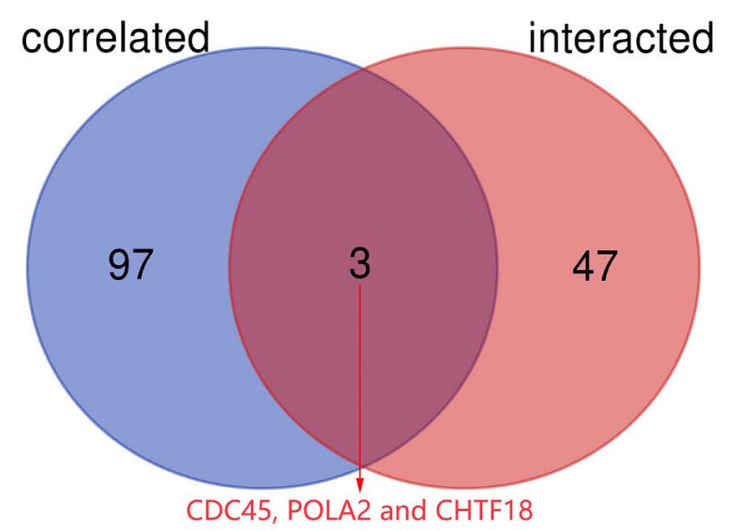

Fig. 7 Genes co-expressed with POLD1 and interaction network of POLD1-binding proteins in HCC tissues. The top 50 significant genes positively (A) and negatively (B) correlated with POLD1. C The volcano plot showing the correlation between POLD1 and genes differentially expressed in HCC. D The chord diagram showing the correlation between POLD1 and POLD molecular family. E Interaction network of 50 POLD1-binding proteins. F An intersection analysis of the POLD1-binding and correlated genes 

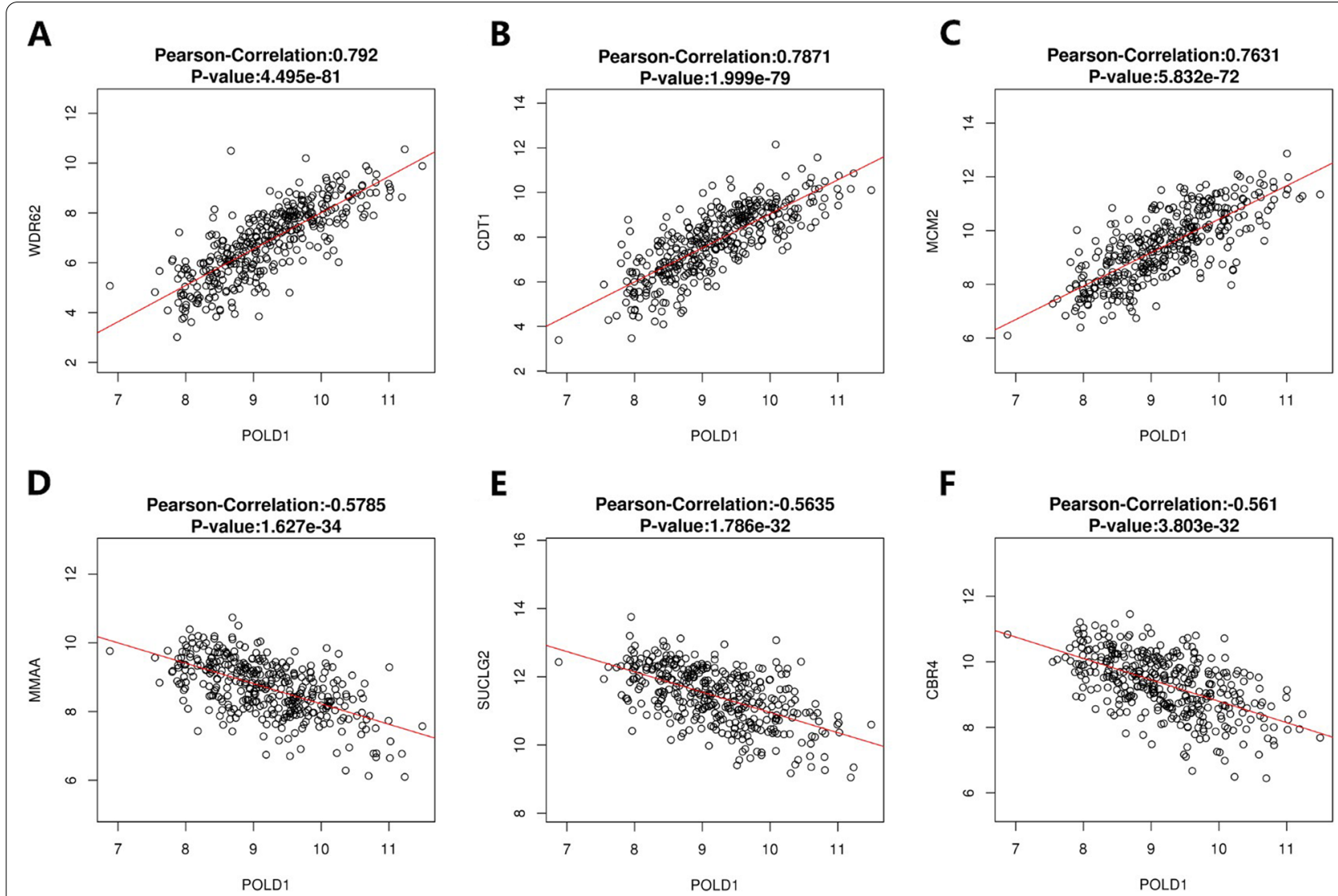

Fig. 8 Correlation analysis between POLD1 mRNA expression and the top three differentially expressed genes in HCC $(n=371)$. A-C The positive correlation between POLD1 and the top three genes (WDR62, CDT1 and MCM2). D-F The negative correlation between POLD1 and the top three genes (MMAA, SUCLG2 and CBR4)

prognostic value of POLD1, were verified using an independent validation cohort from ICGC database.

DNA methylation is the most intensively studied epigenetic mechanism, dysregulated DNA methylation is associated with HCC pathogenesis and progression, and plays role in increased chromosomal instability [34]. While miRNAs are the most well-studied epigenetic regulators in liver cancer, and were reported to be associated with HCC progression and prognosis [34]. Here, we attempted to analyze the mechanisms of the aberrantly expressed POLD1 in HCC and observed that DNA copy gain, low POLD1 methylation, and miR-139-3p downregulation may contribute to POLD1 overexpression. The putative binding site of POLD1 3' UTR by miR-139-3p supported that miR-139-3p might be one of the regulators of POLD1 in HCC. A previous study reported that miR-155 is one of the regulatory miRNAs of POLD1 in a mouse model [35], whereas this finding is not confirmed in HCC by our study (data not shown).

To further identify the underlying mechanisms of POLD1 in HCC carcinogenesis and development, we assessed the association between POLD1 expression and immune cells infiltration. Co-expression genes, PPI network, and functional enrichment analyses of POLD1 in $\mathrm{HCC}$ were also examined. We confirmed that there were significant positive correlations between POLD1 mRNA expression and immune infiltration level of $B$ cell, CD $4+\mathrm{T}$ cell, macrophage, neutrophil and dendritic cell. Moreover, several studies reported that the higher immune infiltration level of dendritic cell, macrophage, and tumor-infiltrating lymphocyte may be predictive biomarkers for ICIs therapy [36, 37]. Furthermore, we identified the top 100 POLD1-correlated genes and 50 experimentally determined POLD1-binding proteins, functional enrichment analysis demonstrated "DNA replication", "mismatch repair" and "cell cycle" pathways might be involved in the effect of POLD1 on HCC pathogenesis. The top three genes (WDR62, CDT1 and MCM2) positively correlated with POLD1 expression play important roles in cell proliferation and apoptosis, and their upregulation has been proved to be associated with tumors development and worse prognosis, such as 


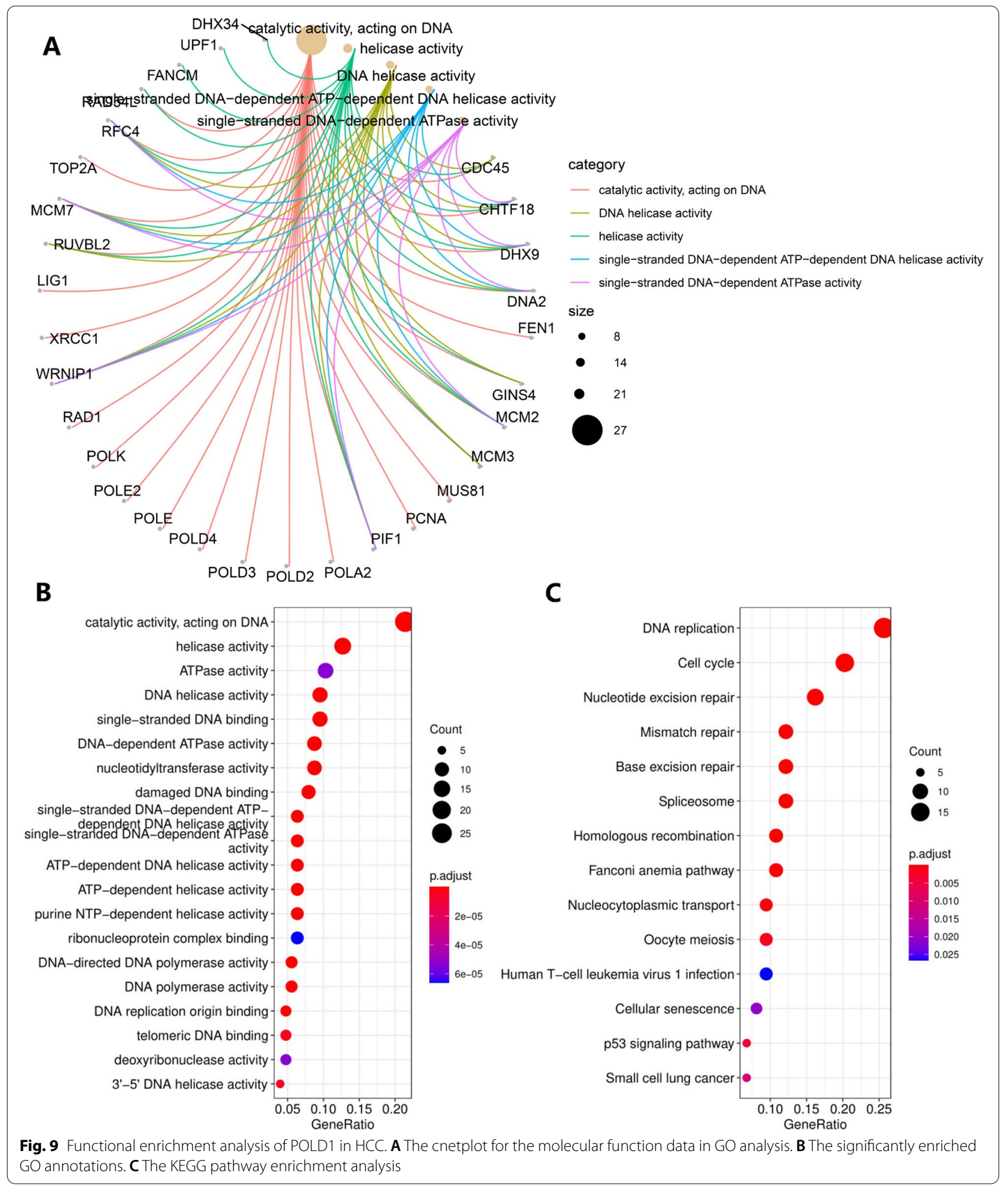

breast cancer and lung adenocarcinoma [38-40]. This is in accordance with the result of the functional enrichment analysis of POLD1 in HCC. However, the role of the top three genes (MMAA, SUCLG2 and CBR4) negatively correlated with POLD1 expression in cancers is not elucidated. 
Unfortunately, we were unable to analyze the effect of POLD1 expression on HCC patients treated with ICIs, because there was no data on HCC patients treated with ICIs in TCGA-LIHC dataset. Besides, we failed to analyze the effect of POLD1 mutation on HCC pathogenesis and patients' survival, because there were only 4 patients with POLD1 mutation among 373 HCC patients in TCGA-LIHC dataset, and no patient carried POLD1 proofreading domain mutation (data not shown).

To verify the prognostic value of POLD1 in patients with various cancer types, Cox analysis and KaplanMeier curves were performed in pan-cancer. The results suggested that POLD1 overexpression was correlated with the poor OS of patients with $\mathrm{ACC}, \mathrm{KICH}$, KIRC, LGG, MESO, PCPG, PRAD, SARC, and HCC. Moreover, POLD1 overexpression was correlated with the poor DFI of patients with PRAD and SARC. As a supplement to previous studies $[9,10]$, our results suggested that POLD1 overexpression was significantly correlated with higher TMB in ACC, BLAC, BRCA, GBM, KICH, LGG, LUAD, LUSC, MESO, OV, PAAD, SARC, SKCM, STAD, TGCT, THCA, UCEC, and UCS. Moreover, POLD1 expression was significantly positively correlated with MSI in ACC, BLCA, BRCA, CESC, HNSC, KICH, KIRC, HCC, LUAD, LUSC, PRAD, SARC, STAD, THCA, and UCEC. These results further confirmed the role of POLD1 in cancer prognosis and genomic instability, and POLD1 could be a promising prognostic marker and potential therapeutic target in various cancers, or biomarker for response to ICIs therapy.

There were still some limitations in our study though we explored the effect and underlying mechanisms of POLD1 in HCC pathogenesis from several different perspectives. First, as mentioned above, we failed to analyze the effect of POLD1 expression on HCC patients treated with ICIs, and the effect of POLD1 mutation on HCC due to its rarity. Second, although the sample size of TCGA-LIHC was relatively large, and the prognostic value of POLD1 was verified using an external validation cohort, further preclinical mechanistic studies and prospective clinical trials are still needed to explore and validate the role of POLD1 in HCC. Several phase 2 clinical trials (such as NCT03461952, NCT02693535, NCT03428802, and NCT03207347) are ongoing to evaluate the efficacy of ICIs or targeted therapies in patients with cancer and POLE/POLD1 mutations. The results of the above clinical trials and more research on the role of POLD1 in cancer carcinogenesis and development would reach more instructive conclusions and contribute to personalized tumor management.

\section{Conclusions}

In conclusion, we found that POLD1 is significantly upregulated in HCC tissue. Overexpression of POLD1 promoted HCC progression potentially through accelerating cell-cycle and improving tolerance of tumor cells to DNA damage. These results help elucidate molecular pathways of HCC carcinogenesis and development. Meanwhile, POLD1 may be a potential prognostic marker and promising therapeutic target in HCC and various cancers.

\section{Abbreviations}

ACC: Adrenocorticalcarcinoma; AFP: Alpha fetoprotein; AUC: Area under the curve; BRCA: Breast invasivecarcinoma; C-index: Concordance index; Cl: Confidence interval; CNA: Copy numberalterations; DFI: Disease-free interval; DLBC: Diffuse large B-celllymphoma; FC: Fold change; GBM: Glioblastoma multiforme; GO: Gene ontology; HCC: Hepatocellularcarcinoma; HNSC: Head and neck squamous cellcarcinoma; HPA: Human Protein Atlas; HR: Hazard ratio; ICGC: International CancerGenome Consortium; ICls: Immune-checkpointinhibitors; IHC: Immunohistochemistry; KEGG: Kyoto encyclopedia ofgenes and genomes; KICH: Kidney chromophobe; KIRC: Kidney clear cellrenal cell carcinoma; LIHC: Liver hepatocellularcarcinoma; LIRI: Liver cancer - RIKEN; LGG: Lower grade glioma; LUAD: Lung adenocarcinoma; LUSC: Lung squamous cellcarcinoma; MESO: Mesothelioma; MSI: Microsatelliteinstability; MSI-H: High MSI.

\section{Supplementary Information}

The online version contains supplementary material available at https://doi. org/10.1186/s12885-022-09284-y.

Additional file 1: Figure S1. (A) Comparison of POLD1 mRNA expression in HCC ( $n=207)$ and in normal liver tissues $(n=175)$ in ICGC-LIRI dataset. Comparison of POLD1 expression in different groups of living status (B), TNM stages (C), and histologic grades (D). (Quasi-likelihood F-test, $P<0.05$ was considered significant, $\left.{ }^{*} P<0.05,{ }^{* *} P<0.01,{ }^{* * *} P<0.001\right)$. Figure S2. Kaplan-Meier curves of overall survival for HCC patients with high and low POLD1 mRNA levels from ICGC-LIRI dataset. Figure S3. Effect of POLD1 expression on prognosis and genomic stability in TCGA pan-cancer cohort. Univariate Cox analysis showed the association between POLD1 expression and overall survival (A) or disease-free interval (B). Correlation between POLD1 expression and TMB (C) or MSI (D). Spearman's correlation coefficients are shown above the bar graphs. (Spearman correlation test, $P<0.05$ was considered significant, ${ }^{*} P<0.05$, ${ }^{* *} P<0.01$, $\left.{ }^{* * *} P<0.001\right)$. Figure S4. Kaplan-Meier curves and log-rank tests for overall survival of patients with high and low POLD1 mRNA levels in adrenocortical carcinoma (A), diffuse large B-cell lymphoma (B), clear cell renal cell carcinoma (C), kidney renal papillary cell carcinoma (D), brain lower grade glioma (E), HCC (F), lung adenocarcinoma (G), mesothelioma (H), sarcoma (I), and uveal melanoma (J). Figure S5. Kaplan-Meier curves and log-rank tests for disease-free interval of patients with high and low POLD1 mRNA levels in prostate adenocarcinoma (A), and sarcoma (B).

\section{Acknowledgements}

We are grateful to all of the reviewers for their valuable comments and suggestions.

\section{Authors' contributions}

HT and TY conceived of and designed the study. HT and TY performed literature search. HT and ZS analyzed the data. HT and TY generated the figures and tables. HT wrote the manuscript, ZS and C B critically reviewed the manuscript. ZS and C B supervised the research. All authors have read and approved the manuscript. 


\section{Funding}

This work was supported by grants from CAMS Innovation Fund for Medical Sciences (No. 2016-12M-1-001).

\section{Availability of data and materials}

The datasets generated and/or analyzed in the current study are available in the TCGA database (http://cancergenome.nih.gov) and the ICGC database (http://icgc.org).

\section{Declarations}

\section{Ethics approval and consent to participate}

Approval was waived by the Medical Ethics Committee of Peking Union Medical College Hospital, as the data retrieved from public databases is publicly available and de-identified.

\section{Consent for publication}

Not applicable.

\section{Conmpeting interests}

No potential conflict of interest was reported by the authors.

Received: 12 May 2021 Accepted: 9 February 2022

Published online: 21 February 2022

\section{References}

1. Villanueva A, Hepatocellular Carcinoma. N Engl J Med. 2019;380(15):1450-62. https://doi.org/10.1056/NEJMra1713263.

2. Meng X, Zhou Y, Zhang S, Lee EY, Frick DN, Lee MY. DNA damage alters DNA polymerase delta to a form that exhibits increased discrimination against modified template bases and mismatched primers. Nucleic Acids Res. 2009;37(2):647-57. doi:https://doi.org/10.1093/nar/gkn1000.

3. Cao B, Zhang Z, Zhang Y, Li J, Liang G, Ling J. Effect of Smilax china L.containing serum on the expression of POLD1 mRNA in human hepatocarcinoma SMMC-7721 cells. Exp Ther Med. 2013;6(4):1070-6. doi:https:// doi.org/10.3892/etm.2013.1264.

4. Qin Q, Tan Q, Li J, Yang W, Lian B, Mo Q, et al. Elevated expression of POLD1 is associated with poor prognosis in breast cancer. Oncol Lett. 2018;16(5):5591-8. doi:https://doi.org/10.3892/ol.2018.9392.

5. Sanefuji K, Taketomi A, Iguchi T, Sugimachi K, Ikegami T, Yamashita Y, et al. Significance of DNA polymerase delta catalytic subunit p125 induced by mutant p53 in the invasive potential of human hepatocellular carcinoma. Oncology. 2010;79(3-4):229-37. doi:https://doi.org/10.1159/000322374.

6. Goldsby RE, Hays LE, Chen X, Olmsted EA, Slayton WB, Spangrude GJ, et al. High incidence of epithelial cancers in mice deficient for DNA polymerase delta proofreading. Proc Natl Acad Sci U S A. 2002;99(24):155605. doi:https://doi.org/10.1073/pnas.232340999.

7. Palles C, Cazier JB, Howarth KM, Domingo E, Jones AM, Broderick P, et al. Germline mutations affecting the proofreading domains of POLE and POLD1 predispose to colorectal adenomas and carcinomas. Nat Genet. 2013;45(2):136-44. doi:https://doi.org/10.1038/ng.2503.

8. Nicolas E, Golemis EA, Arora S. POLD1: Central mediator of DNA replication and repair, and implication in cancer and other pathologies. Gene. 2016;590(1):128-41. doi:https://doi.org/10.1016/j.gene.2016.06.031.

9. He J, Ouyang W, Zhao W, Shao L, Li B, Liu B, et al. Distinctive genomic characteristics in POLE/POLD1-mutant cancers can potentially predict beneficial clinical outcomes in patients who receive immune checkpoint inhibitor. Ann Transl Med. 2021;9(2):129. doi:https://doi.org/10.21037/ atm-20-7553.

10. Wang F, Zhao Q, Wang YN, Jin Y, He MM, Liu ZX, et al. Evaluation of POLE and POLD1 Mutations as Biomarkers for Immunotherapy Outcomes Across Multiple Cancer Types. JAMA Oncol. 2019;5(10):1504-6. doi:https://doi.org/10.1001/jamaoncol.2019.2963.

11. Goldman MJ, Craft B, Hastie M, Repečka K, McDade F, Kamath A et al. Visualizing and interpreting cancer genomics data via the Xena platform. Nat Biotechnol. 2020;38(6):675-8. doi:https://doi.org/10.1038/ s41587-020-0546-8.
12. Tang Z, Kang B, Li C, Chen T, Zhang Z. GEPIA2: an enhanced web server for large-scale expression profiling and interactive analysis. Nucleic Acids Res. 2019;47(W1):W556-60. doi:https://doi.org/10.1093/nar/gkz430.

13. Uhlen M, Zhang C, Lee S, Sjöstedt E, Fagerberg L, Bidkhori G, et al. A pathology atlas of the human cancer transcriptome. Science. 2017;357(6352):eaan2507. doi:https://doi.org/10.1126/science.aan2507.

14. Uhlen M, Oksvold P, Fagerberg L, Lundberg E, Jonasson K, Forsberg M, et al. Towards a knowledge-based Human Protein Atlas. Nat Biotechnol. 2010:28(12):1248-50. doi:https://doi.org/10.1038/nbt1210-1248.

15. Cerami E, Gao J, Dogrusoz U, Gross BE, Sumer SO, Aksoy BA, et al. The cBio cancer genomics portal: an open platform for exploring multidimensional cancer genomics data. Cancer Discov. 2012;2(5):401-4. doi:https:// doi.org/10.1158/2159-8290.Cd-12-0095.

16. Li T, Fan J, Wang B, Traugh N, Chen Q, Liu JS, et al. TIMER: A Web Server for Comprehensive Analysis of Tumor-Infiltrating Immune Cells. Cancer Res. 2017;77(21):e108-10. doi:https://doi.org/10.1158/0008-5472. Can-17-0307.

17. Vasaikar SV, Straub P, Wang J, Zhang B. LinkedOmics: analyzing multiomics data within and across 32 cancer types. Nucleic Acids Res. 2018:46(D1):D956-63. doi:https://doi.org/10.1093/nar/gkx1090.

18. Szklarczyk D, Gable AL, Lyon D, Junge A, Wyder S, Huerta-Cepas J, et al. STRING v11: protein-protein association networks with increased coverage, supporting functional discovery in genome-wide experimental datasets. Nucleic Acids Res. 2019;47(D1):D607-13. doi:https://doi.org/ 10.1093/nar/gky1131.

19. Huang da W, Sherman BT, Lempicki RA. Bioinformatics enrichment tools: paths toward the comprehensive functional analysis of large gene lists. Nucleic Acids Res. 2009;37(1):1-13. doi:https://doi.org/10. 1093/nar/gkn923.

20. Bonneville R, Krook MA, Kautto EA, Miya J, Wing MR, Chen $\mathrm{HZ}$, et al. Landscape of Microsatellite Instability Across 39 Cancer Types. JCO Precis Oncol. 2017;1:1-15. https://doi.org/10.1200/po.17.00073.

21. Paraskevopoulou MD, Georgakilas G, Kostoulas N, Vlachos IS, Vergoulis T, Reczko M. NA-microT web server v5.0: service integration into miRNA functional analysis workflows. Nucleic Acids Res. 2013;41:169-73. https://doi.org/10.1093/nar/gkt393.

22. Bray F, Ferlay J, Soerjomataram I, Siegel RL, Torre LA, Jemal A. Globa cancer statistics 2018: GLOBOCAN estimates of incidence and mortality worldwide for 36 cancers in 185 countries. CA Cancer J Clin. 2018;68(6):394-424. doi:https://doi.org/10.3322/caac.21492.

23. Fuchs J, Cheblal A, Gasser SM. Underappreciated Roles of DNA Polymerase $\delta$ in Replication Stress Survival. Trends Genet. 2021;37(5):47687. doi:https://doi.org/10.1016/j.tig.2020.12.003.

24. Xu Y, Qin Q, Chen R, Wei C, Mo Q. SIRT1 promotes proliferation, migration, and invasion of breast cancer cell line MCF-7 by upregulating DNA polymerase delta1 (POLD1). Biochem Biophys Res Commun. 2018;502(3):351-7. doi:https://doi.org/10.1016/j.bbrc.2018.05.164.

25. Cheng AL, Hsu C, Chan SL, Choo SP, Kudo M. Challenges of combination therapy with immune checkpoint inhibitors for hepatocellular carcinoma. J Hepatol. 2020;72(2):307-19. doi:https://doi.org/10.1016/j. jhep.2019.09.025.

26. Samstein RM, Lee $\mathrm{CH}$, Shoushtari AN, Hellmann MD, Shen $\mathrm{R}$, Janjigian $Y Y$, et al. Tumor mutational load predicts survival after immunotherapy across multiple cancer types. Nat Genet. 2019;51(2):202-6. doi:https:// doi.org/10.1038/s41588-018-0312-8.

27. Petrelli F, Ghidini M, Ghidini A, Tomasello G. Outcomes Following Immune Checkpoint Inhibitor Treatment of Patients With Microsatellite Instability-High Cancers: A Systematic Review and Meta-analysis. JAMA Oncol. 2020;6(7):1068-71. doi:https://doi.org/10.1001/jamaoncol.2020. 1046.

28. Cui Y, Keles S, Charbonnier LM, Jule AM, Henderson L, Celik SC, et al. Combined immunodeficiency caused by a loss-of-function mutation in DNA polymerase delta 1. J Allergy Clin Immunol. 2020;145(1):391-401. doi:https://doi.org/10.1016/j.jaci.2019.10.004.

29. Tumini E, Barroso S, Calero CP, Aguilera A. Roles of human POLD1 and POLD3 in genome stability. Sci Rep. 2016;6:38873. doi:https://doi.org/ 10.1038 /srep38873.

30. Song J, Hong P, Liu C, Zhang Y, Wang J, Wang P. Human POLD1 modulates cell cycle progression and DNA damage repair. BMC Biochem. 2015;16:14. doi:https://doi.org/10.1186/s12858-015-0044-7. 
31. Kaelin WG Jr. The concept of synthetic lethality in the context of anticancer therapy. Nat Rev Cancer. 2005;5(9):689-98. https://doi.org/10. 1038/nrc1691.

32. Job A, Tatura M, Schäfer C, Lutz V, Schneider H, Lankat-Buttgereit B, et al. The POLD1 (R689W) variant increases the sensitivity of colorectal cancer cells to ATR and CHK1 inhibitors. Sci Rep. 2020;10(1):18924. doi:https://doi.org/10.1038/s41598-020-76033-1.

33. Hocke S, Guo Y, Job A, Orth M, Ziesch A, Lauber K, et al. A synthetic lethal screen identifies ATR-inhibition as a novel therapeutic approach for POLD1-deficient cancers. Oncotarget. 2016;7(6):7080-95. doi:https://doi.org/10.18632/oncotarget.6857.

34. Toh TB, Lim JJ, Chow EK. Epigenetics of hepatocellular carcinoma. Clin Transl Med. 2019;8(1):13. doi:https://doi.org/10.1186/ s40169-019-0230-0.

35. Czochor JR, Sulkowski P, Glazer PM. miR-155 Overexpression Promotes Genomic Instability by Reducing High-fidelity Polymerase Delta Expression and Activating Error-Prone DSB Repair. Mol Cancer Res. 2016;14(4):363-73. doi:https://doi.org/10.1158/1541-7786.Mcr-15-0399.

36. Gibney GT, Weiner LM, Atkins MB. Predictive biomarkers for checkpoint inhibitor-based immunotherapy. Lancet Oncol. 2016;17(12):e542-51. doi:https://doi.org/10.1016/s1470-2045(16)30406-5.

37. Baba Y, Nomoto D, Okadome K, Ishimoto T, Iwatsuki M, Miyamoto Y, et al. Tumor immune microenvironment and immune checkpoint inhibitors in esophageal squamous cell carcinoma. Cancer Sci. 2020;111(9):3132-41. doi:https://doi.org/10.1111/cas.14541.

38. Yousef EM, Furrer D, Laperriere DL, Tahir MR, Mader S, Diorio C, et al. MCM2: An alternative to Ki-67 for measuring breast cancer cell proliferation. Mod Pathol. 2017;30(5):682-97. doi:https://doi.org/10.1038/modpa thol.2016.231.

39. Pozo PN, Cook JG. Regulation and Function of Cdt1; A Key Factor in Cell Proliferation and Genome Stability. Genes. 2016;8(1):2. doi:https://doi.org/ 10.3390/genes8010002.

40. Bu Y, Zhang L, Ma X, Wang R, Zhang X, Li J. Systematic Analysis of the Oncogenic Role of WDR62 in Human Tumors. Dis Markers. 2021;2021:9940274. doi:https://doi.org/10.1155/2021/9940274.

\section{Publisher's Note}

Springer Nature remains neutral with regard to jurisdictional claims in published maps and institutional affiliations.

Ready to submit your research? Choose BMC and benefit from:

- fast, convenient online submission

- thorough peer review by experienced researchers in your field

- rapid publication on acceptance

- support for research data, including large and complex data types

- gold Open Access which fosters wider collaboration and increased citations

- maximum visibility for your research: over 100M website views per year

At BMC, research is always in progress.

Learn more biomedcentral.com/submissions 